Chapter 4

\title{
One Stage Allogenic Acellular Dermal Matrices (ADM) and Split-Thickness Skin Graft with Negative Pressure Wound Therapy
}

\author{
Hyunsuk Suh and Joon Pio Hong \\ Additional information is available at the end of the chapter \\ http://dx.doi.org/10.5772/53304
}

\section{Introduction}

After first success of epidermal autotransplantation onto a granulating wound in 1869, skin graft become a standard option for closing defect that cannot be closed primarily. [1] Skin graft can be used in various clinical situations. For the reconstruction of traumatic wounds, burn wound, soft tissue defect or skin defect after cancer ablation surgery or after removal of pigmented skin lesion, diabetic wounds and after scar contracture release and pigmented scar removal, skin graft are widely used around the world.

\subsection{Skin graft types}

\subsubsection{Split-thickness skin graft or full-thickness skin grafts}

Skin grafts are generally classified as either split-thickness or full-thickness grafts. Splitthickness skin graft includes varying amounts of dermis whereas a full-thickness graft includes the entire dermis. The amount of dermis within the graft determines the likelihood of survival and the degree of contracture. Split-thickness grafts can survive in conditions with less vascularity, but they have a greater likelihood of contracture. In contrast, full-thickness grafts require a better vascular bed for survival but undergo less contracture. Contracture involves contraction of a healed graft and is caused by myofibroblast activity. The thinner the skin graft is, the greater the contracture of the graft. Granulating wounds or skin defect left to heal secondarily, without any grafting, will eventually demonstrate the greatest degree of contracture and are most prone to hypertrophic scarring. [2] The success of skin graft depends on the ability of the grafted skin to receive nutrients in first few days and, subse- 
quently, vascular ingrowth from the recipient bed. Because the full-thickness skin graft is thicker, survival of the graft demands more well-vascularized bed.

The ultimate goal of skin grafting is first to achieve aesthetic results similar to the adjacent recipient site in terms of color, texture and thickness of skin with minimal contractures. Second is to achieve complete "take" of graft, which is closely related to minimal scar tissue formation. The third goal is to make minimal scar on the donor site. [3]

The full-thickness skin graft has superior result than split-thickness skin graft in aesthetic aspects. It has minimal contractures. We can achieve best esthetic results by doing the fullthickness skin graft for all kinds of full-thickness skin defects if there is unlimited donor sites and complete graft "take". But donor site for the full-thickness skin harvest is limited. The size of full-thickness skin is also limited. Even if there is unlimited supply of full-thickness skins, we cannot use it for all kinds of recipient beds because full-thickness graft demands more perfusion from the recipient bed. For these reasons, split-thickness skin grafts are commonly used for most of large skin defects. But the major disadvantage to traditional split-thickness skin graft is related to donor site morbidity, including permanent scar formation. Unfortunately, the thicker the harvested split-thickness skin, the more donor site morbidity that results. These issues are compounded in those with thin skin such as the young patients and in those with limited donor sites that will require serial reharvesting of each donor site after epithelialization. In cases in which grafted sites are frequently exposed, one may consider the aesthetic limitations of a split-thickness skin graft to be less favorable compared with thicker graft.

\section{Allogenic dermis with skin graft}

To mimic a full-thickness graft and to minimize donor site morbidity, the addition of an allogenic acellular dermal matrix (ADM) to the split-thickness graft can be considered. The use of STSG combined with a layer of allogenic ADM underneath provides an additional layer of tissue coverage and the potential benefit of promoting the imbibition phase of wound healing.

A vascular linkage to allogenic AMD is achieved within 3 days after transplantation as compared with 2 to 3 weeks observed in alternative materials that are manufactured from animal skin. Accordingly, in cases in which the autograft segment was transplanted simultaneously, biointegration is achieved with a vascular linkage on the third day. [4] allogenic ADM has been used in a wide range of areas including soft tissue extensions for cosmetic purposes, skin grafts for patients with burns and replacement of tympanic membrane, nasal septum, and dura mater. Recent studies have shown that application of allogenic $\mathrm{ADM}$ is cosmetically and functionally excellent compared with conventional split thickness skin graft. This is because it is effective in maintaining texture and elasticity of the skin tissue by enhancing the underlying dermis. [5-7] When used as a graft, it is repopulated and revascularized by the recipient's cells and supports the survival of an overlying split-thickness skin graft. [5] 


\section{Why allogenic dermis?}

Many acellular dermal substitutes are used clinically. The 3D matrices should enable progressive vascularization and fibroblast invasion from the surrounding tissues. Bovine collagen and porcine collagen are used especially in wound treatment and skin reconstruction. Nevertheless, porcine collagen is regaining interest, with as big advantage the absence of Prion diseases, the porcine viruses also needed to be considered, and porcine collagen might elicit more foreign body reactions than bovine collagen. The animal acellular dermal matrix is commercialized as MatriDerm consists of collagen (bovine dermis) coated with elastin hydrolysate from the ligamentum nuchae. But MatriDerm seems to degrade sooner after 3 months, and no statistical evidence of long-term clinical effectiveness after 1 year. [8,9]

Histological analysis of the underlying allograft derrnal matrix after composite allodermis and skin graft revealed that there were infiltration of host fibroblasts and neovascularization into the allodermis in the absence of an inflammatory cell infiltrate. There were minimal histological differences noted between the allodermis and the dermal component of the control skin graft. Electron-microscopic analysis on day 16 postgraft demonstrates the presence of an intact basement membrane at the junction of the allograft derrnal matrix, similar to that seen on the dermal component of the control site STSG. Keratinocytes had migrated across and attached to the basement membrane, as shown by the presence of hemidesmosomes. Collagen fibers showed typical ultrastructural characteristics, as defined by fiber diameter, banding and orientation. Most importantly, the processed dermal matrix remains and supports the infiltration of host fibroblasts with typical morphological characteristics of normal synthetic fibroblasts. [5]

These findings suggest that the supplemental dermis supplied by the allogenic acellular dermal matrix, which remains and works as matrix, can potentially improve the healing characteristics of an autograft. The clinically relevant implication is the potential for closure of an extensively burned patient using minimal autograft skin, but resulting in a skin cover whose quality is superior to that typically obtained with thin autografts with their tendency for scarring and contracture.

\subsection{Human donor skins for dermal grafting}

Human acellular dermal matrices (ADM) are derived from human dermis, treated to remove all immunogenic elements: keratinocytes, fibroblasts, vascular endothelium, and smooth muscle cells. Virus screening is also obliged. However, several different technical methods for processing those matrix have been developed, [10,11,12] all aiming to preserve the integrity of the remaining dermal elements as good as possible. The main elements of all $\mathrm{ADM}$ are the collagen and elastin fibers, which serve as a 3D natural matrix for the invasion of the native cellular elements in vivo. The amount of remaining growth factors, cytokines depends on the processing method. The first ADM were processed by trypsin, $[10,13]$ freeze-thawing, [11,12] or long incubations with enzymes. Most of those matrix remained highly antigenic, which lead to poor graft survival. $[10,11,12,13,14,15]$ Several different products are currently available for wound care. 
1. AlloDerm ${ }^{\circledR}$ (Lifecell Corp., Branchburg, NJ) is an acellular de-epidermalized dermis product that is a semipermanent skin substitute. It is a cryopreserved and lyophilized allodermis that acts strictly as a dermal replacement. It has no protective epidermal analogue. AlloDerm ${ }^{\circledR}$ does become incorporated into the host by tissue ingrowth. AlloDerm ${ }^{\circledR}$ is a freeze-dried cryopreserved acellular dermal matrix on an intact basement membrane complex obtained by processing human donor skin in a saline solution (sodium dodecyl sulfate) and enzymes. [5,15-19] It is decellularized, freeze-dried, and biochemically stabilized, and has been successful alone and in combination with cultured autografts (two-steps procedure) in the treatment of burn wounds and dermal defects. Additionally, AlloDerm ${ }^{\circledR}$ is procured by cryopreservation which may affect the integrity of the elastin matrix, and its manufacturing is expensive.

2. DermaMatrix ${ }^{\mathrm{TM}}$ (Synthes, Inc., West Chester, PA) $[20,21]$ is human donor skin processed using a combination of detergent and acid washes and is then freeze dried. It is especially commercialized for reconstructive surgery, but clinical studies in wound care remain to be published.

3. GlyaDerm ${ }^{\mathrm{TM}}$ (Euro Skin Bank, Netherlands) is another acellular dermal collagen-elastin matrix, obtained by the treatment of glycerolized human donor skin with a low concentration of $\mathrm{NaOH}$. The elastin matrix is not damaged by this manufacturing and preser vation method, which should lead to a more durable effect. [22,23] Additional advantages of glycerol preservation include inactivation of viruses and ease of storage and handling. GlyaDerm is provided by a non-governmental, non-profit organization, the Euro Skin Bank (the Netherlands) and is intended to be cost-effective, enabling widespread application.

4. SureDerm ${ }^{\mathrm{TM}}$ (Hans Biomed Corp. South Korea) is obtained by sequential treatments with dispase followed by Triton X-100. [24] The enzymatic treatment with dispase removes the epidermal layer. It is freeze-dried and stored at temperatures of $2^{\circ} \mathrm{C}$ to $8^{\circ} \mathrm{C}$. SureDerm can be applied together with an one step split thickness skin graft, but there is a high risk of infection. Histologic examination showed that this product is completely absorbed within 4 months. [24]

5. CGDerm ${ }^{\mathrm{TM}}$ (Daewoong Bio Corp., Seoul Korea) is also an allograft derived from human skin. The epidermis and dermis are removed from the subcutaneous layer of the skin during the recovery procedure. The tissue is then processed using a sodium chloride solution and detergent to remove the epidermis and all viable dermal cells while maintaining the original dermal collagen matrix. The cells are removed to minimize inflammation or immunorejection at the surgical site. CGDerm ${ }^{\mathrm{TM}}$ is then treated in a disinfection solution that combines detergents with acidic and antiseptic reagents to further clean the tissue for sterility. Finally, it is freeze-dried, cut to size and packaged in a terminally sterilized double pouch and envelope. [25]

In a clinical report, an ADM with ultrathin autograft composite inhibited contraction and improved long-term cosmesis in patients with major burns. [26,27] A one-staged composite 
dermal and epidermal replacement was also reported with processed cadaver dermis and thin autograft for acute burn reconstructions. [28] Combined with AlloDerm® and a splitthickness skin graft, it can mimic a full-thickness skin graft providing a favorable long-term result. Thin autogenous skin graft or cultured keratocytes can be used over the ADM to provide permanent coverage. [27]

\section{Clinical reports of ADM and split-thickness skin grafts}

\subsection{ADM and autograft for full thickness burn patients}

The need to replace skin lost through injury is particularly crucial in extensively burned patients with limited donor site availability. Scar contracture with hypertrophy or joint contracture is common after 1:3 more meshed split thickness graftings. In full thickness burn patients, dermal component of ADM can prevent and reduce joint contracture and scar formation after skin graft surgery. Dermal component also reduce trans-epidermal water loss and erythema value than split thickness skin graft only group. [29] In the clinical report they used 1:3 6 meshed autograft as one-stage reconstruction method. [29] And in other reports comparing non-ADM graft only with 1:1.5 meshed skin graft and ADM graft with 1:1.5 mesh auto graft, skin elasticity was twice as high with ADM group in post operative day 60 and superior cosmesis without hypertrophic scarring in postoperative day 90. [5]

\subsection{ADM and autograft for full-thickness scalp defects}

In scalp wound patients which the calvaria is exposed, the use of flap is generally considered. However, in cases in which patients' general status is poor or vascular insufficiency is present, the use of flap becomes difficult. Treatment involved removal of the outer table of the skull and application of acellular human dermis (AlloDerm $\left.{ }^{\circledR}\right)$. Then split-thickness skin graft was performed in a single phase split-thickness skin graft was performed in a single step. As compared with the flap,the use of AlloDerm ${ }^{\circledR}$ was technically simple and less timeconsuming due to it being a single-step procedure. It is therefore effective in shortening the treatment period and securing excellent treatment outcomes. [30]

\section{Problems using ADM and autograft}

However, this process can be cumbersome when processed in single stage as a result of the need for serum imbibition and revascularization of separate layers of graft. So, a longer period of stabilization is required for revascularization to occur and to minimize the risk of graft failure. It is more true with unmeshed skin graft. 


\section{What is NPWT (Negative pressure wound therapy)?}

Negative pressure wound therapy is a well-established form of treatment to enhance wound healing. It was first introduced by Argenta and Morykwas in 1997 and it has been used in a variety of conditions to reduce the size of wounds. [31,32] The mechanical force of negative pressure on wounds is known to augment local blood flow, reduce interstitial edema, reduce bacterial count, help retract the edges of the wound, and possibly affect the cellular activity and angiogenesis of the wound. [31-33,34] These factors together result in effective granulation and epithelization, successfully accelerating the healing of chronic wounds often seen in plastic and reconstructive, thoracic, orthopedic, and general surgical cases. [31,32,35-38]

\subsection{NPWT for skin graft}

The concept of using negative pressure wound therapy (NPWT) to secure a skin graft is not new. NPWT has been used instead of traditional dressing methods including bolster dressing or tie-over dressing. This technique provides near perfect contact between the graft and the recipient bed with a pressure equivalent to the negative pressure applied even on a complex curved anatomic areas. [39,40] NPWT minimizes shearing between the graft and wound bed and prevent formation of fluid and seroma also to promote proper contact between the graft and the recipient bed. Moreover, it may remove blood and exudates by negative pressure, minimizing the risk of hematoma as well as the risk of infection. [41] Improved microcirculation and increased tissue oxygen concentration also provide a desirable environment for graft survival. [42] NPWT also can be used to maximize graft take of full-thickness skin graft. [43] In treating the donor site of a radial forearm flap, Avery et al showed improved graft take without the need of splinting the arm, thereby reducing the morbidities and the length of hospital stay. [44]

Skin grafts typically fail because of shearing force over the graft skin or the development of a haematoma, seroma or infection underneath it. [45,46] The application of negative pressure contours the dressing materal so that it conforms to the wound surface. This stabilises the graft and helps prevent shearing and reduces the risk of haematoma and seroma formation, while helping to prevent contamination. Increased granulation facilitates revascularisation and attachment of the graft to the wound bed [35,36]. Numerous clinical studies have shown the successful use of NPWT in the management of skin and biomatrix grafts. $[41,42,44,47,48]$

A blinded, randomised controlled trial (RCT) investigated the effects of NPWT compared with standard bolster dressings over split-thickness skin grafts. Results showed that qualitative graft take was significantly better with NPWT as compared to standard bolster dressing $(P<0$ 05). NPWT over STSG improved the quality of the graft's appearance postoperatively, which may increase patient satisfaction. [49]

10-years retrospective review of 142 patients treated with an STSG in foot and ankle reconstructive surgeries also showed the effect of negative pressure wound therapy over skin 
graft. [48] Wound types included pressure, post-traumatic, and diabetic wounds, benign tumors, osteomyelitis, and other chronic ulcers. STSG patients received either NPWT $(n=87)$ or conventional therapy $(n=55)$ dressing. The results showed a significant difference in graft acceptance between the NPWT and conventional therapy groups. There were significantly fewer repeated graft and fewer complications, such as seroma, haematoma and infection, were observed

\section{Negative pressure wound therapy for AlloDerm ${ }^{\circledR}-$ split-thickness skin graft}

A retrospective study reports the use of negative pressure wound dressing with a simultaneous Acellular dermal matrix-split-thickness skin graft in resurfacing full-thickness defects and evaluates the efficacy over conventional tieover grafts. [50] A prospective study of 47 cases of skin defects treated by 1-stage allodermis and a split-thickness skin graft with NPWT showed that $97.8 \%$ graft take was noted at day 5 and the mean time until complete healing was 5.8 days. Statistically significant graft take (day 5$)$ and time until complete healing was noted $(\mathrm{P}<0.05)$. Good aesthetic and functional result mimicking a full-thickness skin graft was achieved. [50]

\subsection{One-stage graft saves time and effort}

Two-stage operation is common for wound coverage with ADM and autogenous skin graft. In most cases, use of acellular dermal matrix is followed by definitive coverage with skin graft at the second stage after the "take" of acellular dermal matix. [51] Negative pressure wound therapy make two-step procedure a single-stage operation. Negative pressure wound therapy over "composite of AMD and split-thickness skin" stabilizes the graft and remove hematoma or seroma under the skin and ADM. And it also facilitates revascularisation and attachment of the graft to the wound bed [35,36]. One-stage graft saves time and efforts of surgeon and patients by reducing operation time and dressing change.

\section{Clinical applications}

One-stage ADM and split-thickness skin graft can be applied to re-surface every types of wound with skin loss due to numerous etiologies including acute full thickness burns, acute trauma, chronic wounds, soft tissue defect with granulating bed and donor site of flap surgery which cannot be closed primarily.

\subsection{Clinical cases}

\subsubsection{Case 1}

A 71-year-old diabetic male was suffered from a diabetic foot. He had chronic renal failure on peritoneal dialysis. One month after percutaneous transluminal angioplasty of occlusions 
of superficial femoral artery, CGDerm ${ }^{\mathrm{TM}}$ and split thickness skin graft was done. After composite tissue graft, CuraVac ${ }^{\circledR}(400-600 \mu \mathrm{m}$ pore size, Daewoong Pharm. CO. LTD., Seoul Korea) and portable suction device Curasys ${ }^{\mathrm{TM}}$ which provides cyclonic suction mode was applied for 10 days. Graft remain soft without any hypertrophic scar after 6 months. (Fig 1)
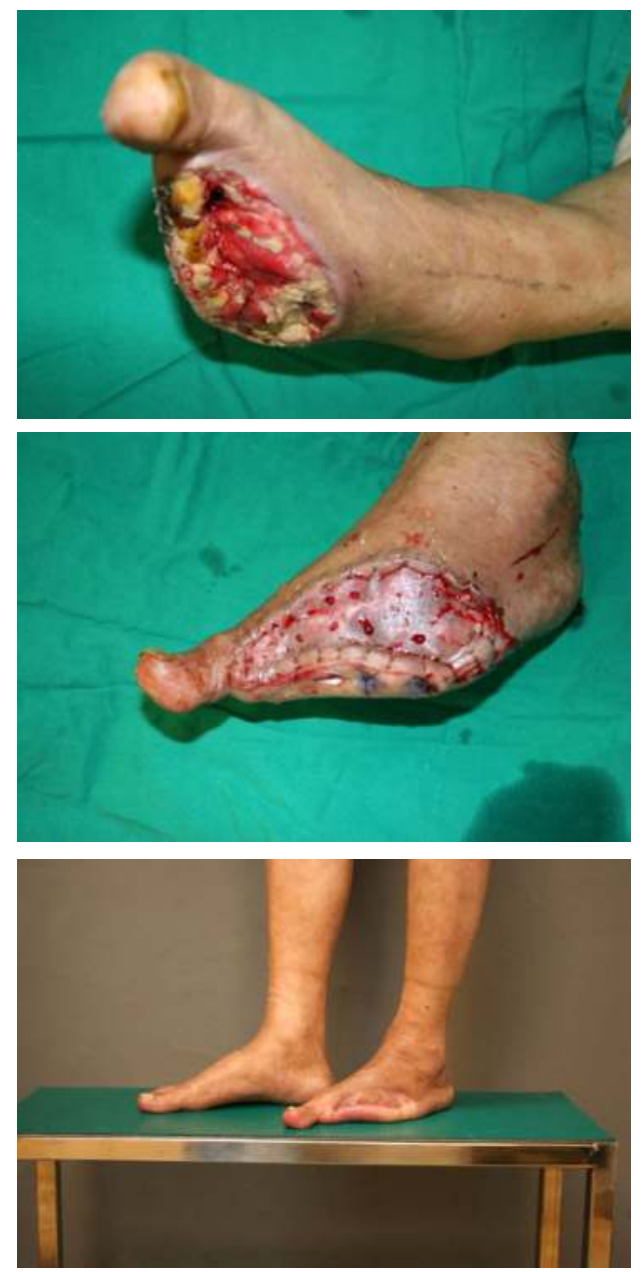

Figure 1. (Above) Before application of graft (Center) Just after grafting of CGDerm ${ }^{\mathrm{TM}}$ over granulating tissue (Below) 6 month following CGDerm ${ }^{\mathrm{TM}}$, split thickness skin graft and negative pressure wound therapy. After composite tissue graft, CuraVac ${ }^{\circledast}$ (400-600 $\mu$ m pore size, Daewoong Pharm. CO. LTD., Seoul Korea) and portable suction device Curasys $^{\top M}$ (Daewoong Pharm. CO. LTD., Seoul Korea) which provides cyclonic suction mode was applied for 10 days. 


\subsubsection{Case 2}

A 74-year-old female was suffered from diabetic foot. After serial debridement, CGDerm ${ }^{\mathrm{TM}}$ and split thickness skin graft was done. After composite tissue graft, CuraVac ${ }^{\circledR}(400-600 \mu \mathrm{m}$ pore size, Daewoong Pharm. CO. LTD., Seoul Korea) and portable suction device Curasys ${ }^{\mathrm{TM}}$ which provides cyclonic suction mode was applied for postoperative5 days. Graft maintained soft with minimal marginal hypertrophy. (Fig. 2)

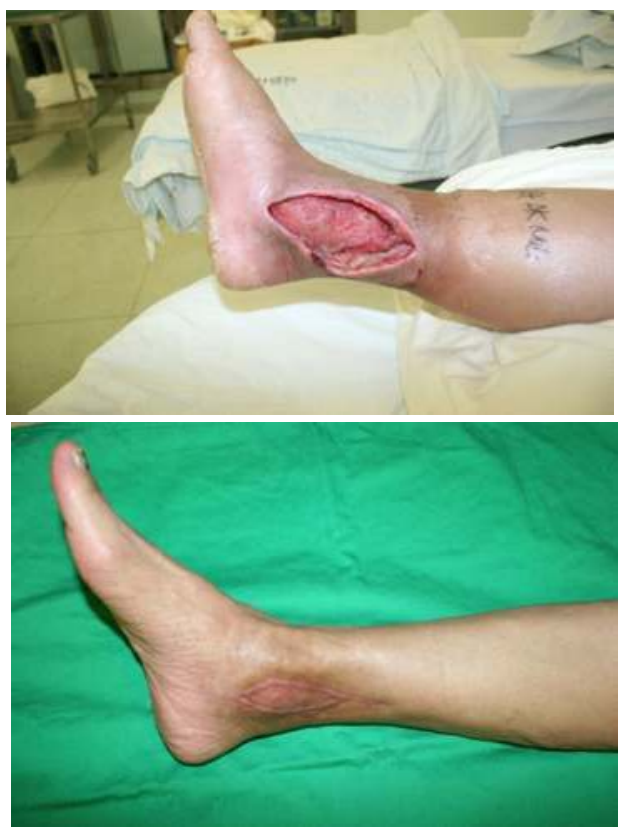

Figure 2. (Above) After debridement (Below) 2 months after CGDerm ${ }^{\mathrm{TM}}$, split thickness skin graft and negative pressure wound therapy. Wound remained soft without skin breakdown.

\subsubsection{Case 3}

A 60-year-old male had tonsil cancer. He underwent reconstruction with radial forearm free flap for oral cavity soft tissue defect. And donor site of radial forearm flap donor site was closed with CGDerm ${ }^{\mathrm{TM}}$, split thickness skin graft and negative pressure wound therapy. Graft remained soft without any ulceration. (Fig3) 

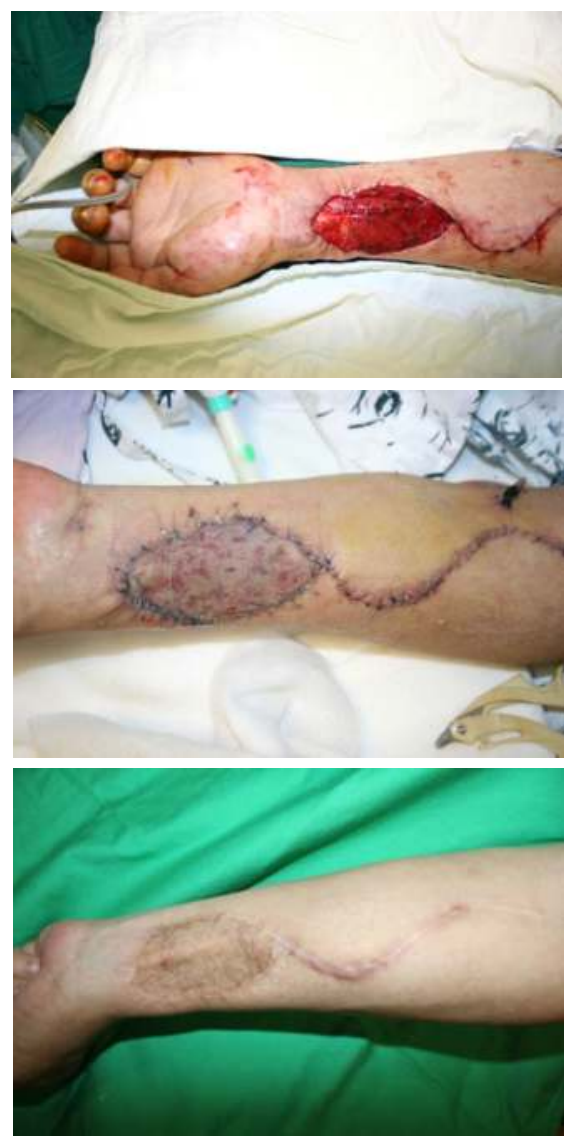

Figure 3. (Above) Before applying human acellular dermal matrix(ADM) (Center) 5 days after CGDerm ${ }^{\mathrm{TM}}$, split thickness skin graft and negative pressure wound therapy with CuraVac ${ }^{\circledR}$ and portable suction device Curasys ${ }^{\mathrm{TM}}$. Simple dressing was applied after removal of first negative pressure dressing. (Below) 2 years after skin graft

\subsubsection{Case 4}

A 57-year-old female underlying diabetes had necrotising fasciitis after consumption of seafood in overseas travel. She underwent serial debridement and CGDerm ${ }^{\mathrm{TM}}$, meshed split thickness skin graft and negative pressure wound therapy with CuraVac ${ }^{\circledR}$ was done. Wound remain soft without any hypertrophy after 8 months. (Fig 4.) 

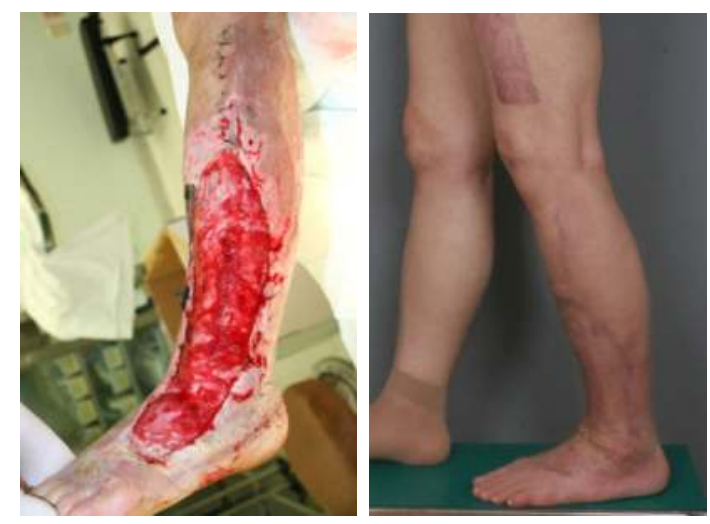

Figure 4. Left) After debridement and before skin grafting (Right) 8 month after AlloDerm ${ }^{\circledast}$ (Lifecell Corp., Branchburg, NJ) and thin split thickness skin graft. Wound remain soft without hypertrophy and ulceration.

\section{Protocols}

After adequate debridement of the wound bed, full range of the defect was covered with graftable ADM with 8 13/1000 inch thickness and fixed with minimal absorbable sutures after aseptical rehydrating of the implants with normal saline. Split-thickness skin graft was harvested from thigh or buttock region with a thickness of 8 12/1000 inches and a few slit incisions were made to promote removal of any fluid or blood. Skin was then sutured with non-absorbable suture or staplers to fix the graft to the bed and margin of the wound. Once the graft has been placed, a sterile open-cell, polyurethane ether foam containing PVC connection tubing is applied over the composite of AMD and split-thickness skin and sealed with occlusive dressing. A continuous or intermittent or cyclic negative pressure from $75 \mathrm{mmHg}$ to $125 \mathrm{mmHg}$ can be delivered by wall suction facilities or special suction device. Negative wound therapy was maintained for first 5 days and on the first opening of the wound, negative pressure dressing was stopped and minimal wound dressing was perform if needed. On day 5, you can confirm nearly complete take of composite tissue by checking adherence of graft and capillary refills. Additional dressing can be applied base on the needs.

\section{Conclusion}

Split-thickness skin added to acellular dermal matrix provides a sufficient amount of dermis to prevent contracture and promote better aesthetic outcome, and the negative pressure therapy ensured fast and complete take of the 2-layered composite graft. This option can be 
used to achieve healing mimicking a full-thickness skin graft without requiring large fullthickness donor sites.

\section{Disclosure}

The. senior author holds a co-patent for cyclic negative pressure wound therapy system and has given the rights to Daewoong Pharmaceutical Company.

\section{Author details}

Hyunsuk Suh and Joon Pio Hong

Department of Plastic Surgery, Seoul Asan Medical Center, University of Ulsan College of Medicine, Seoul, Korea

\section{References}

[1] JL. R. Greffes epidermiques. Bulletin de la Societe Imperiale de Chirurgie de Paris. 1869;10:51.

[2] Ragnell A. The secondary contracting tendency of free skin grafts; an experimental investigation on animals. Br J Plast Surg. 1952;5:6-24.

[3] Corps BV. The effect of graft thickness, donor site and graft bed on graft shrinkage in the hooded rat. Br J Plast Surg. 1969;22:125-133.

[4] Soejima K, Chen X, Nozaki M, Hori K, Sakurai H, Takeuchi M. Novel application method of artificial dermis: one-step grafting procedure of artificial dermis and skin, rat experimental study. Burns. 2006;32:312-318.

[5] Wainwright DJ. Use of an acellular allograft dermal matrix (AlloDerm) in the management of full-thickness burns. Burns. 1995;21:243-248.

[6] Costantino PD, Wolpoe ME, Govindaraj S, et al. Human dural replacement with acellular dermis: clinical results and a review of the literature. Head \& neck. 2000;22:765-771.

[7] Kridel RW, Foda H, Lunde KC. Septal perforation repair with acellular human dermal allograft. Archives of otolaryngology--head \& neck surgery. 1998;124:73-78.

[8] Bloemen MC, van Leeuwen MC, van Vucht NE, van Zuijlen PP, Middelkoop E. Dermal substitution in acute burns and reconstructive surgery: a 12-year follow-up. Plastic and reconstructive surgery. 2010;125:1450-1459. 
[9] van Zuijlen PP, Vloemans JF, van Trier AJ, et al. Dermal substitution in acute burns and reconstructive surgery: a subjective and objective long-term follow-up. Plastic and reconstructive surgery. 2001;108:1938-1946.

[10] Oliver RF, Grant RA, Kent CM. The fate of cutaneously and subcutaneously implanted trypsin purified dermal collagen in the pig. British journal of experimental pathology. 1972;53:540-549.

[11] Fang CH, Robb EC, Yu GS, Alexander JW, Warden GD. Observations on stability and contraction of composite skin grafts: xenodermis or allodermis with an isograft overlay. The Journal of burn care \& rehabilitation. 1990;11:538-542.

[12] Grillo HC, McKhann CF. The Acceptance and Evolution of Dermal Homografts Freed of Viable Cells. Transplantation. 1964;2:48-59.

[13] Oliver RF, Barker H, Cooke A, Stephen L. 3H-collagen turnover in non-cross-linked and aldehyde-cross-linked dermal collagen grafts. British journal of experimental pathology. 1982;63:13-17.

[14] Oliver RF, Barker H, Cooke A, Grant RA. Dermal collagen implants. Biomaterials. 1982;3:38-40.

[15] Walter RJ, Matsuda T, Reyes HM, Walter JM, Hanumadass M. Characterization of acellular dermal matrices (ADMs) prepared by two different methods. Burns. 1998;24:104-113.

[16] Jones I, Currie L, Martin R. A guide to biological skin substitutes. Br J Plast Surg. 2002;55:185-193.

[17] Kirsner RS, Falanga V, Eaglstein WH. The development of bioengineered skin. Trends in biotechnology. 1998;16:246-249.

[18] Livesey SA, Herndon DN, Hollyoak MA, Atkinson YH, Nag A. Transplanted acellular allograft dermal matrix. Potential as a template for the reconstruction of viable dermis. Transplantation. 1995;60:1-9.

[19] Rennekampff HO, Hansbrough JF, Woods V, Jr., Kiessig V. Integrin and matrix molecule expression in cultured skin replacements. The Journal of burn care \& rehabilitation. 1996;17:213-221.

[20] Becker S, Saint-Cyr M, Wong C, et al. AlloDerm versus DermaMatrix in immediate expander-based breast reconstruction: a preliminary comparison of complication profiles and material compliance. Plastic and reconstructive surgery. 2009;123:1-6; discussion 107-108.

[21] Shores JT, Gabriel A, Gupta S. Skin substitutes and alternatives: a review. Advances in skin \& wound care. 2007;20:493-508; quiz 509-410.

[22] Pirayesh A, Dur AH, Paauw NJ, et al. Evaluation of acellular dermis for closure of abdominal wall defects in a rat model. European surgical research. Europaische chirurgische Forschung. Recherches chirurgicales europeennes. 2008;41:346-352. 
[23] Richters CD, Pirayesh A, Hoeksema H, et al. Development of a dermal matrix from glycerol preserved allogeneic skin. Cell and tissue banking. 2008;9:309-315.

[24] Takami Y, Matsuda T, Yoshitake M, Hanumadass M, Walter RJ. Dispase/detergent treated dermal matrix as a dermal substitute. Burns. 1996;22:182-190.

[25] Product information of CGDerm.

[26] Tsai CC, Lin SD, Lai CS, Lin TM. The use of composite acellular allodermis-ultrathin autograft on joint area in major burn patients--one year follow-up. Kaohsiung J Med Sci. 1999;15:651-658.

[27] Wainwright D, Madden M, Luterman A, et al. Clinical evaluation of an acellular allograft dermal matrix in full-thickness burns. The Journal of burn care \& rehabilitation. 1996;17:124-136.

[28] Callcut RA, Schurr MJ, Sloan M, Faucher LD. Clinical experience with Alloderm: a one-staged composite dermal/epidermal replacement utilizing processed cadaver dermis and thin autografts. Burns. 2006;32:583-588.

[29] Yim H, Cho YS, Seo CH, et al. The use of AlloDerm on major burn patients: AlloDerm prevents post-burn joint contracture. Burns. 2010;36:322-328.

[30] Jung SN, Chung JW, Yim YM, Kwon H. One-stage skin grafting of the exposed skull with acellular human dermis (AlloDerm). The Journal of craniofacial surgery. 2008;19:1660-1662.

[31] Argenta LC, Morykwas MJ. Vacuum-assisted closure: a new method for wound control and treatment: clinical experience. Ann Plast Surg. 1997;38:563-576; discussion 577.

[32] Morykwas MJ, Argenta LC, Shelton-Brown EI, McGuirt W. Vacuum-assisted closure: a new method for wound control and treatment: animal studies and basic foundation. Ann Plast Surg. 1997;38:553-562.

[33] Jones SM, Banwell PE, Shakespeare PG. Advances in wound healing: topical negative pressure therapy. Postgraduate medical journal. 2005;81:353-357.

[34] Venturi ML, Attinger CE, Mesbahi AN, Hess CL, Graw KS. Mechanisms and clinical applications of the vacuum-assisted closure (VAC) Device: a review. American journal of clinical dermatology. 2005;6:185-194.

[35] Stone PA, Hass SM, Flaherty SK, DeLuca JA, Lucente FC, Kusminsky RE. Vacuumassisted fascial closure for patients with abdominal trauma. The Journal of trauma. 2004;57:1082-1086.

[36] Tang AT, Okri SK, Haw MP. Vacuum-assisted closure to treat deep sternal wound infection following cardiac surgery. Journal of wound care. 2000;9:229-230. 
[37] DeFranzo AJ, Marks MW, Argenta LC, Genecov DG. Vacuum-assisted closure for the treatment of degloving injuries. Plastic and reconstructive surgery. 1999;104:2145-2148.

[38] O'Connor J, Kells A, Henry S, Scalea T. Vacuum-assisted closure for the treatment of complex chest wounds. The Annals of thoracic surgery. 2005;79:1196-1200.

[39] Nakayama Y, Iino T, Soeda S. A new method for the dressing of free skin grafts. Plastic and reconstructive surgery. 1990;86:1216-1219.

[40] Stokes TH, Follmar KE, Silverstein AD, et al. Use of negative-pressure dressings and split-thickness skin grafts following penile shaft reduction and reduction scrotoplasty in the management of penoscrotal elephantiasis. Ann Plast Surg. 2006;56:649-653.

[41] Blackburn JH, 2nd, Boemi L, Hall WW, et al. Negative-pressure dressings as a bolster for skin grafts. Ann Plast Surg. 1998;40:453-457.

[42] Isago T, Nozaki M, Kikuchi Y, Honda T, Nakazawa H. Skin graft fixation with negative-pressure dressings. The Journal of dermatology. 2003;30:673-678.

[43] Landau AG, Hudson DA, Adams K, Geldenhuys S, Pienaar C. Full-thickness skin grafts: maximizing graft take using negative pressure dressings to prepare the graft bed. Ann Plast Surg. 2008;60:661-666.

[44] Avery C, Pereira J, Moody A, Gargiulo M, Whitworth I. Negative pressure wound dressing of the radial forearm donor site. International journal of oral and maxillofacial surgery. 2000;29:198-200.

[45] Hanasono MM, Skoracki RJ. Securing skin grafts to microvascular free flaps using the vacuum-assisted closure (VAC) device. Ann Plast Surg. 2007;58:573-576.

[46] Scherer LA, Shiver S, Chang M, Meredith JW, Owings JT. The vacuum assisted closure device: a method of securing skin grafts and improving graft survival. Arch Surg. 2002;137:930-933; discussion 933-934.

[47] Ward C, Ciraulo D, Coulter M, Desjardins S, Liaw L, Peterson S. Does treatment of split-thickness skin grafts with negative-pressure wound therapy improve tissue markers of wound healing in a porcine experimental model? The journal of trauma and acute care surgery. 2012;73:447-451.

[48] Blume PA, Key JJ, Thakor P, Thakor S, Sumpio B. Retrospective evaluation of clinical outcomes in subjects with split-thickness skin graft: comparing V.A.C.(R) therapy and conventional therapy in foot and ankle reconstructive surgeries. International wound journal. 2010;7:480-487.

[49] Moisidis E, Heath T, Boorer C, Ho K, Deva AK. A prospective, blinded, randomized, controlled clinical trial of topical negative pressure use in skin grafting. Plastic and reconstructive surgery. 2004;114:917-922.

[50] Kim EK, Hong JP. Efficacy of negative pressure therapy to enhance take of 1-stage allodermis and a split-thickness graft. Ann Plast Surg. 2007;58:536-540. 
[51] Askari M, Cohen MJ, Grossman PH, Kulber DA. The use of acellular dermal matrix in release of burn contracture scars in the hand. Plastic and reconstructive surgery. 2011;127:1593-1599. 